\title{
VOLUMES OF SMALL BALLS ON OPEN MANIFOLDS: LOWER BOUNDS AND EXAMPLES
}

\author{
CHRISTOPHER B. CROKE AND HERMANN KARCHER
}

\begin{abstract}
Question: "Under what curvature assumptions on a complete open manifold is the volume of balls of a fixed radius bounded below independent of the center point?" Two theorems establish such assumptions and two examples sharply limit their weakening. In particular we give an example of a metric on $\mathbf{R}^{4}$ (extending to higher dimensions) of positive Ricci curvature, whose sectional curvatures decay to 0 , and such that the volume of balls goes uniformly to 0 as the center goes to infinity.
\end{abstract}

0. Introduction. The following question came up in discussions of the first author with Peter Li:

Under what curvature assumptions on a complete open manifold $M$ can one find a constant $c(M, R)$ such that the volume of balls $B(x, R)$ can be bounded from below independent of the midpoint $x$,

$$
\operatorname{vol}(B(x, R)) \geq c(M, R) ?
$$

Note that in the presence of a lower bound on the Ricci curvature one needs to prove the above inequality only for some $R$ since the Bishop-Gromov volume comparison theorem $[\mathbf{G r}$, p. 124] then gives a bound $c(M, r)$ for $r<R$. Note also that a lower bound on the injectivity radius implies the above inequality [Cr, Proposition 14]: If $r<\operatorname{inj}(M)$ then $\operatorname{vol}(B(x, r)) \geq c(n) \cdot r^{n}$. In dimension 2 , Theorem $\mathrm{A}$ below is a satisfying positive answer since the assumptions do not imply a lower bound on the injectivity radius while any weakening of the assumptions leads to immediate counterexamples.

THEOREM A. If $M^{2}$ is complete with curvature $K \geq 0$ then there exist $c(M)$ such that for radii $R \leq 1$ the following holds:

$$
\operatorname{area}(B(x, R)) \geq c(M) \cdot R^{2} .
$$

In higher dimensions however we have

EXAMPLE 1. There are convex hypersurfaces $M^{d}$ in $\mathbf{R}^{d+1}$ such that for $d \geq 3$ no lower bounds as in (0.1) exist for $M^{d}$.

In dimensions $>2$ we therefore assume an upper curvature bound. But then one gets uniform control even on the injectivity radius. The following theorem has been rediscovered several times.

Received by the editors August 10, 1987.

1980 Mathematics Subject Classification (1985 Revision). Primary 53C20; Secondary 53A07.

Research supported by NSF Grant MCS 79-01780 and the Sloan foundation. Both authors were supported by invitations to the I.H.E.S. and the M.S.R.I. 
THEOREM (TOPONOGOV [BZ, p. 17]). If $M$ is open, complete and $0<K \leq$ $\Delta$ then (in contrast to the compact case) $\operatorname{inj}(M) \geq \pi \cdot \Delta^{-1 / 2}$.

This follows from the Gromoll-Meyer exhaustion by compact totally convex sets since these do not contain closed geodesics [GM], and nearest nonconjugate cutpoints of $q$ are midpoints of geodesic loops based at $q$.

It is lesser known that the curvature assumption can be further weakened:

THEOREM I (SHARAFUTDINOV [Sh]). If $M$ is open, complete and $0 \leq K \leq \Delta$ and if $\operatorname{inj}(S)$ is the injectivity radius of any Cheeger-Gromoll soul $S$ of $M$ then

$$
\operatorname{inj}(M) \geq \min \left(\operatorname{inj}(S), \pi \cdot \Delta^{-1 / 2}\right) .
$$

Sharafutdinov's proof is based on the following useful fact: For the totally convex exhaustion $\left\{C_{t}\right\}$ there is a length nonincreasing deformation from $C_{t}$ to $C_{\tau}(t>\tau)$. This also implies that any two souls are isometric. Unfortunately he postponed the proof twice, we could not find his final reference and Croke-Schroeder were discouraged from writing up their version after learning about Sharafutdinov's paper. We include a new short proof of Theorem I.

The above theorems and examples are our answer to the question $(0.1)$ in the case of nonnegative curvature. Clearly one cannot relax sectional curvature assumptions to $-b^{2} \leq K \leq \Delta$ without adding something else. There are two results which give some hope that the assumption Ric $>0$ might provide that additional help:

ThEOREM (BISHOP-GROMOV [Gr]). Let $M^{n}$ be complete with Ric $\geq 0$. Fix $x_{0} \in M$ and $R_{0} \in \mathbf{R}$. Then

$$
\operatorname{vol}\left(B\left(x, R_{0}\right)\right) \geq \operatorname{const}\left(M, x_{0}, R_{0}\right) \cdot d\left(x, x_{0}\right)^{-n} .
$$

THEOREM (CALABI-YAU). Let $M$ be noncompact, complete and Ric $\geq 0$. Fix $x_{0} \in M$. Then

$$
\operatorname{vol}\left(B\left(x_{0}, R\right)\right) \geq \text { const } \cdot R \text {. }
$$

The first theorem says that the volume of balls of radius $R_{0}$ cannot decrease too fast as the center $x$ is moved out. The second theorem (which is proved with the same ideas: consider the rays from some unit ball $B$ to some point at $\infty$ and view $B$ from distance $2,4,6, \ldots$; one gets disjoint sets of not smaller volume) says that the volume of balls grows (at least linearly) with the radius. Nevertheless, in dimensions $\geq 4$ the assumption Ric $>0$ is of no help, not even when combined with curvatures which get small outside compact sets. In the following Example 2 there are not just occasionally small balls as in Example 1, but to any given $c(M, R)$ there is a compact set such that outside of it all balls of radius $R$ have volume $\leq c(M, R)$.

EXAMPLE 2. There is a complete metric on $\mathbf{R}^{4}$ with the following properties:

(a) Ric $>0$.

(b) The sectional curvatures at $x \in \mathbf{R}^{4}$ are bounded in absolute value by const $\cdot d\left(x, x_{0}\right)^{-2+\delta}$.

(c) For every $\varepsilon>0$

$$
\lim _{r \rightarrow \infty}(\sup \{\operatorname{vol}(B(x, \varepsilon)) ; d(x, 0) \geq r\})=0 .
$$

The example extends to higher dimensions. The construction works with any $0<$ $\delta<\frac{2}{3}$. We do not know whether Ric $>0$ might help in dimension 3 . 
1. Proof of Theorem I. We follow the steps of the Cheeger-Gromoll construction of a continuous exhaustion of $M$ by compact totally convex sets [CG].

(a) First they obtain "large" compact totally convex sets $C_{t}, t_{0} \leq t<\infty$ using complements of horoballs of rays. This is continued with the inner parallel sets

$$
C_{t}=\left\{p \in C_{t_{0}} ; d\left(p, \partial C_{t_{0}}\right) \geq t_{0}-t\right\}, \quad t_{1} \leq t \leq t_{0},
$$

up to the set $C_{t_{1}}$ at maximal distance from $\partial C_{t_{0}}$. Corresponding to this continuous exhaustion we have the continuous nonincreasing function

$$
g:\left[t_{1}, \infty\right) \rightarrow \mathbf{R}, \quad g(t):=\min \left\{\operatorname{inj}(p) ; p \in C_{t}\right\} .
$$

The essential part of our proof shows the

Claim. $g(t)<\pi \cdot \Delta^{-1 / 2}$ implies for the left derivative: $g_{l}^{\prime}(t)=0$. An easy generalization of the mean value theorem to continuous functions with left derivatives then gives $g(t)$ cannot drop below $\min \left\{\operatorname{inj}\left(C_{t_{1}}\right), \pi \cdot \Delta^{1 / 2}\right\}$. This is already a weak form of Theorem I; as said below (0.1) it implies a lower bound for volumes of balls.

(b) The structure theorem in [CG] says that $C_{t_{1}}$ is a compact totally convex set in a lower dimensional totally geodesic submanifold of $M$. If $C_{t_{1}}$ has a boundary in this submanifold the inner parallel sets can be continued; this is repeated until the soul $S$ (a totally convex geodesic submanifold without boundary) is reached. Our proof of the claim is also repeated at each step, proving Theorem I as stated.

Proof of the claim. Either $g$ is constant on $(t-\delta, t]$ and there is nothing to prove-or there is some $q \in \partial C_{t}$ with $g(t)=\operatorname{inj}(q)<\pi \cdot \Delta^{-1 / 2}$. A (nonconjugate) nearest cut point $\bar{q}$ of $q$ determines a geodesic loop $\gamma$ based at $q$ and $\gamma$ is in $C_{t}$ by total convexity. Hence also inj $(\bar{q})=g(t)$ so that the angle of $\gamma$ at $q$ cannot be $<\pi$. The closed geodesic $\gamma$ cannot meet $C_{\tau}$ for $\tau<t$ by total convexity of $C_{\tau}$. Note $\operatorname{inj}(\gamma(s))=\operatorname{inj}(q)$.

Next choose some shortest geodesic $c$ from $\gamma \subset \partial C_{t}$ to the farthest set $C_{t_{1}}$ and rename $q=c(0)$. For small $s$ (to avoid conjugate points) there are at least two geodesics $\gamma_{1}, \gamma_{2}$ from $c(s)$ to $\bar{q}$ opposite to $q$ on $\gamma$, and we can choose $\gamma_{1}, \gamma_{2}$ to depend differentiably on $s$. Then

(i) $f(s):=\frac{1}{2}$ (length $\left(\gamma_{1}\right)+$ length $\left.\left(\gamma_{2}\right)\right) \geq \operatorname{inj}(c(s))$,

(ii) $f^{\prime}(0)=0\left(f<\pi \cdot \Delta^{-1 / 2}\right.$ is differentiable and $\left.c^{\prime}(0) \perp \gamma\right)$.

Since $g$ is nonincreasing, (i) and (ii) imply $g_{l}^{\prime}(t)=0$ as soon as we know $c(s) \in$ $C_{t \text {-const.s }}$ for some const $>0$. Such a constant does not exist uniformly, considering the inner parallel sets of the lense-shaped intersection of two balls. But, at least, $c(s)$ is for $s>0$ in the interior of $C_{t}$. Therefore, picking some $0<\sigma<\frac{1}{2} g(t)$, we have $c(\sigma) \in C_{t-\varepsilon}, \varepsilon>0$. We shall bound the opening angle $\varphi$ of the geodesic cone with vertex $q$, axis $c$ and base the disc of radius $\varepsilon$ perpendicular to $c$ at $c(\sigma)$ by $\cos \varphi \leq\left(1+\varepsilon^{2} \sigma^{-2}\right)^{-1 / 2}$. Since the cone is in $C_{t}$ this angle estimate gives the const $>$ 0 and finishes the proof. The hypotenuse $h$ of a right triangle in $M$ with edges $\varepsilon, \sigma$ is bounded from above by comparing with the flat plane: $h^{2} \leq \sigma^{2}+\varepsilon^{2}$, and from below by comparing with the sphere of curvature $\Delta: h \geq \sigma$. Toponogov's theorem gives for the angle $\varphi$ opposite $\varepsilon: \cos \varphi \leq\left(h^{2}+\sigma^{2}-\varepsilon^{2}\right) / 2 h \sigma \leq\left(1+\varepsilon^{2} \sigma^{-2}\right)^{-1 / 2}$.

\section{Example 1.}

Recall. Under the assumptions of Theorem A no lower bound for the injectivity radius can be deduced: Add a sequence of disjoint tangential cones to a paraboloid of revolution, then change the cones slightly to obtain a hypersurface with $K>0$ 


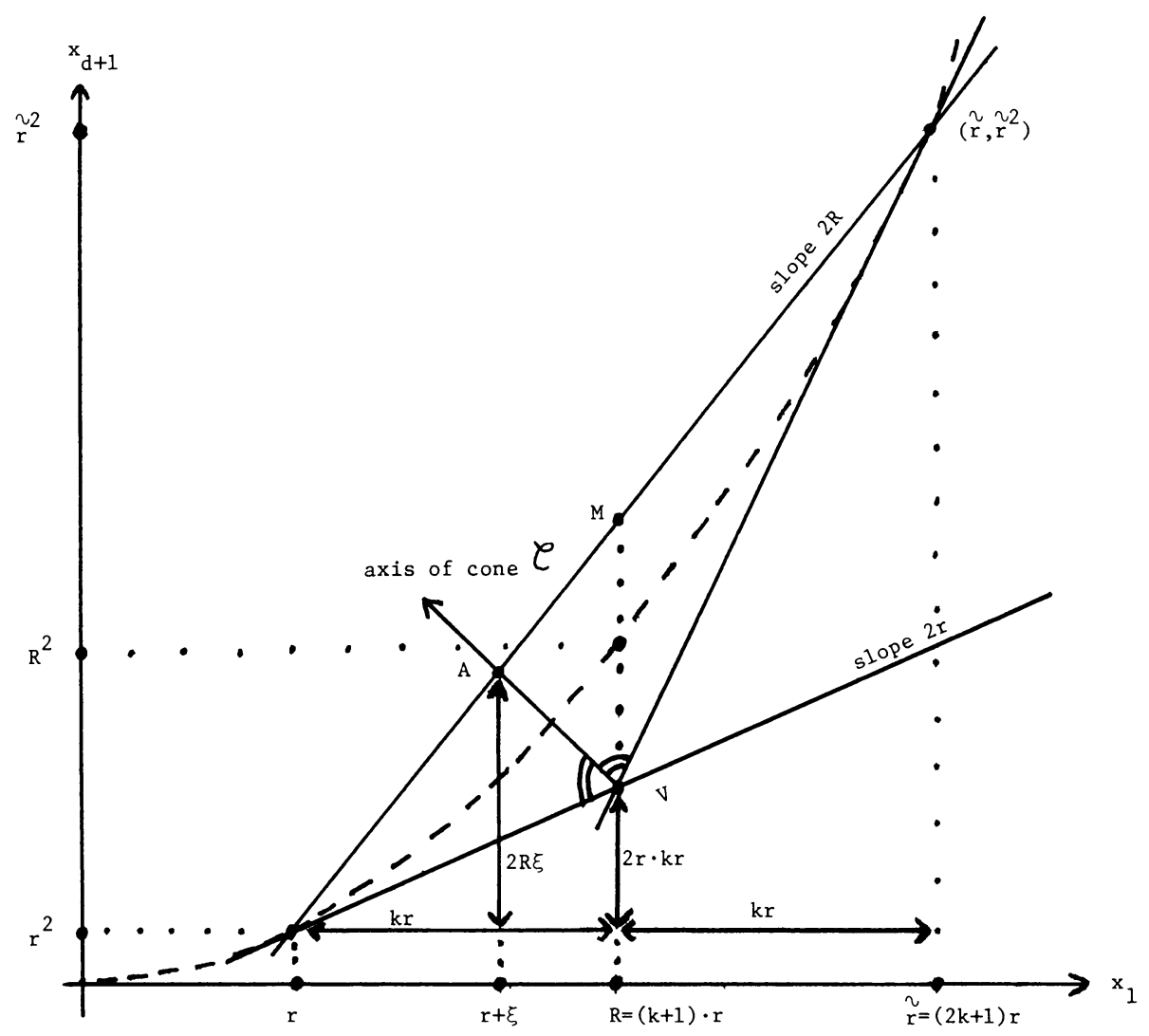

Figure. Paraboloid $\mathscr{P}$ of revolution and tangential cone $\mathscr{C}$ intersected with $x_{1}-x_{d+1}$-plane. The "axis" is the angle bisector of the two generators of the cone $\mathscr{C}$ in this plane.

and conical singularities. The injectivity radius function is bounded above by the distance to the nearest singularity. Therefore one can smooth so that $\inf _{M}$ inj-rad remains zero.

To obtain an example where no bound as in (0.1) exists we attach a more carefully controlled sequence of disjoint tangential cones to the paraboloid of revolution

$$
\mathscr{P}:=\left\{x \in \mathbf{R}^{d+1} ; x_{d+1}=x_{1}^{2}+\cdots+x_{d}^{2}\right\} .
$$

The volume of the balls around the vertices of the sequence of cones goes to zero if $d \geq 3$. (Clearly one can modify the example first to $K>0$ and then smooth the singularities.) The desired sequence of cones can be chosen inductively because of the

Claim. Let $\mathscr{C}$ be the tangential cone (to the above paraboloid) which has its vertex at $V:=\left((k+1) \cdot r, 0, \ldots, 0,(2 k+1) r^{2}\right)$ (see Figure). Assume $r \geq 1, k \geq 5$. Then

(i) The lowest point of $\mathscr{P} \cap \mathscr{C}$ is $\left(r, 0, \ldots, 0, r^{2}\right)$, the highest point is $\left(\tilde{r}, 0, \ldots, \tilde{r}^{2}\right)$ with $\tilde{r}=(2 k+1) r$. 
(ii) Distance $(V, \mathscr{P}) \geq \frac{1}{2}|M-V| \cdot 1 / 2 R=k^{2} r /(2 k+2)$.

(iii) If $\varepsilon \leq k^{2} r /(2 k+2)$ then $\operatorname{vol}_{d}(B(V, \varepsilon))=1 / d \cdot \omega \cdot \varepsilon^{d}$ where

$$
\omega:=\operatorname{vol}_{d-1}(\mathscr{C} \cap\{x ;|x-V|=1\}) \text {. }
$$

(iv) $\omega$ is bounded by the volume $\tilde{\omega}$ of a spherical spindle of opening angle $\vartheta$ :

$$
\omega \leq \tilde{\omega}=\sin ^{d-2}(\vartheta) \cdot \operatorname{vol}\left(S^{d-2}\right) \cdot \int_{-\pi / 2}^{\pi / 2} \cos ^{d-2}(\rho) d \rho .
$$

(v) The opening angle can be made arbitrarily small: $\tan ^{2} \vartheta \leq 6 / k$.

(vi) $\operatorname{vol}_{d}(B(V, \varepsilon) \cap \mathscr{C}) \leq \operatorname{const}(d) \cdot k^{-(d-2) / 2} \cdot \varepsilon^{d}$.

PROOF. (i) and (ii) are immediate properties of $\mathscr{P}$, (see Figure). (iii) follows from (ii) since the formula gives the volume of balls on euclidean cones. (iv) follows since the convex spherical spindle which we are going to describe contains the spherical convex set $\mathscr{C} \cap\{x ;|x-V|=1\}$ and therefore has larger boundary volume. (v) is straightforward once we have the spindle and (vi) summarizes (iii)-(v).

Description of the spherical spindle. The cone $\mathscr{C}$ touches the paraboloid $\mathscr{P}$ along an ellipsoid with one "large" axis in the $x_{1}-x_{d+1}$-plane and $(d-2)$ "short" axis perpendicular to the $x_{1}-x_{d+1}$-plane. We do not consider the ray from the vertex $V$ to the midpoint $M$ of this ellipsoid as the axis of the cone $\mathscr{C}$ but the angle bisector of the two generators of $\mathscr{C}$ in the $x_{1}-x_{d+1}$-plane. The hyperplane which contains this axis and is orthogonal to the $x_{1}-x_{d+1}$-plane we call the equator plane of $\mathscr{C}$; it intersects $\mathscr{C}$ in a $(d-1)$-dimensional cone of revolution whose opening angle we denote by $\vartheta$. We enlarge $\mathscr{C}$ to a cone $\tilde{\mathscr{C}}$, by taking the convex hull of the equator section of $\mathscr{C}$ and of the line through $V$ perpendicular to the equator plane.

The intersection of $\tilde{\mathscr{E}}$ with the unit sphere is the desired spherical spindle. In terms of spherical geometry the spindle is the double cone over the $(d-2)$ dimensional small sphere of radius $\vartheta$ in the equator plane and the vertices of this double cone are the two poles of the equator. The spherical volume of the spindle is trivially given by the formula in (iv) while the formula for $\omega$ is much more complicated.

The estimate (v) of the opening angle $\vartheta$ :

Let $A$ be a point on $\mathscr{P}$ where a generator of $\mathscr{C}$ in the equator plane touches; the opening angle $\vartheta$ is the angle between the generator $V A$ and the axis. We write

$$
A=\left(r+\xi, a, r^{2}+2 R \cdot \xi\right), \quad a \in R^{d-1} .
$$

Then

$$
A \in \mathscr{P} \leftrightarrow|a|^{2}=r^{2}+2 R \xi-(r+\xi)^{2}=\xi \cdot(2 k r-\xi)
$$

and (see (v) above)

$$
\begin{aligned}
\tan ^{2} \vartheta & =|a|^{2} \cdot\left((k r-\xi)^{2}+\left(2 R \xi-2 k r^{2}\right)^{2}\right)^{-1} \\
& \leq \xi \cdot 2 k r \cdot(k r-\xi)^{-2}
\end{aligned}
$$

In the $x_{1}-x_{d+1}$-plane we have for the axis of $\mathscr{C}$

|slope $\mid \leq 1 \quad$ (slope of lowest generator is $2 r \geq 1$ )

which implies an upper bound $L$ for $\xi$ given by

$$
2 R \cdot L-1 \cdot(k r-L)=2 r \cdot k r \quad \text { (see Figure) }
$$


or

$$
L=k r \cdot \frac{1+2 r}{1+(2 k+2) r}<\frac{3}{2 k+2} \cdot k r
$$

Finally

$$
\max _{\{\xi \leq L\}} 2 k r \xi \cdot(k r-\xi)^{-2} \leq \frac{3}{k+1}\left(1-\frac{3}{2 k+2}\right)^{-2}<\frac{6}{k}
$$

completes the estimate of $\tan ^{2} \vartheta$.

3. Proof of Theorem A. A. D. Alexandrow has shown $[\mathbf{A 1}$, p. 325] that any complete Riemannian metric on $\mathbf{R}^{2}$ with $K \geq 0$ ("positive Krümmung" in the German edition means $K \geq 0$ ) can be isometrically embedded as the boundary of a closed convex domain $D$ in $\mathbf{R}^{3}$. Although we have an intrinsic proof of Theorem A which (not surprisingly) is simpler than Alexandrow's embedding construction we nevertheless chose to use this isometric embedding (a) for brevity and (b) to show in terms of euclidean geometry how the constant of Theorem A comes about.

(i) If $M^{2}$ has more than one end then $M^{2}$ contains a line which splits $M^{2}$ as an isometric product, i.e. $M^{2}$ is isometric to a flat cylinder and we even have a lower bound for the injectivity radius in this case.

(ii) If $M^{2}$ is nonorientable, then its soul must be a homotopically nontrivial closed geodesic, i.e. $M^{2}$ is a Möbius-band, its twofold cover has 2 ends and is the flat cylinder of (i). A flat Möbius band again has a lower bound for its injectivity radius.

(iii) We are left with the case where the soul of $M^{2}$ is a point, i.e. $M^{2}$ is diffeomorphic to $\mathbf{R}^{2}$; Alexandrow's theorem applies, and the convex set $D$ which it gives has nonempty interior (since $M^{2}$ is not flat).

For any $x \in D$ consider the closed convex cone $C$ of halflines from $x$ in $D . C$ is nonempty, unique up to parallel translation (in $D$ ) and $C$ contains no line. Thus we can pick $v_{0} \in C$ such that $\left\langle v, v_{0}\right\rangle>0$ for all $0 \neq v \in C$. We take $v_{0}$ as the direction of the positive $z$-axis and the support plane of $D$ perpendicular to $v_{0}$ as the plane $z=0$. Consider for $t>0$ the closed convex curves $\gamma_{t}:=\partial D \cap\{z=t\}$ (recall $\stackrel{\circ}{D} \neq \varnothing$ ). The orthogonal projection of $\gamma_{t^{\prime}}$ into the $x-y$-plane contains the projection of $\gamma_{t}$ for $t^{\prime}>t$, hence $L\left(\gamma_{t^{\prime}}\right) \geq L\left(\gamma_{t}\right)$. Put $L:=L\left(\gamma_{1}\right)$ and $\varepsilon_{0}:=\frac{1}{2} L$.

In the compact set $M_{c}:=\partial D \cap\{z \leq 1\}$ we have a positive lower bound for the injectivity radius and an upper bound for the curvature and therefore a constant $c\left(M_{c}\right)$ such that

$$
\operatorname{vol}(B(x, \varepsilon)) \geq c \cdot \varepsilon^{2} \quad \text { for } x \in M_{c}, \varepsilon \leq \varepsilon_{0}
$$

To deal with the noncompact portion $M_{\infty}:=\partial D \cap\{z \geq 1\}$ we consider through each $p \in M_{\infty}$ also the "meridian" $\sigma_{p}$ which is defined as the intersection of $M_{B}$ with the half plane spanned by $p$ and the $z$-axis. After parametrising each $\sigma_{p}$ by arclength $s$ it follows first from convexity that on each meridian the derivative $\partial z / \partial s$ is increasing and then from compactness of $\gamma_{1}$ that there exists $A>0$ such that $d z / d s \geq A$ uniformly on the meridians (which we only consider in $\{z \geq 1\}$ ). For $p \in M_{\infty}$ (denote its $z$-coordinate by $z_{p}$ ) and for $\varepsilon \leq \varepsilon_{0}$ we can now estimate 
using the coarea formula:

$$
\begin{aligned}
\operatorname{Area}(B(p, \varepsilon)) & \geq \int_{t \geq z_{p}} L(B(p, \varepsilon) \cap\{z=t\}) d t \\
& \geq \int_{z_{p}}^{z_{p}+\varepsilon A} L\left(B(p, \varepsilon) \cap \gamma_{t}\right) d t
\end{aligned}
$$

Finally, since $L\left(\gamma_{t}\right) \geq 2 \varepsilon$ we have first from the triangle inequality and then from the triangle inequality and then from $d z / d s \geq A$ :

$$
\begin{aligned}
L\left(B(p, \varepsilon) \cap \gamma_{t}\right) & \geq 2\left(\varepsilon \text {-length }\left(\sigma_{p} \cap\left\{z_{p} \leq z \leq t\right\}\right)\right) \\
& \geq 2\left(\varepsilon-\left(t-z_{p}\right) / A\right) .
\end{aligned}
$$

This gives the desired bound $\operatorname{Area}(B(p, \varepsilon)) \geq A \cdot \varepsilon^{2}$.

4. Example 2. The example roughly looks as follows: Change the radial distances from a point $0 \in \mathbf{C} P^{2}$ to throw its cutlocus to $\infty$; simultaneously change the scale on the distance spheres to force the length of the Hopf fibres to converge to zero while keeping Ric $>0$. We will quote what we need about the distance spheres of $\mathrm{C} P^{2}$ and derive in (4.1)-(4.9) what we want to know about our metric on $\mathbf{R}^{4}$ (see end of Introduction).

The failure for a bound as in (0.1) to exist can neither be blamed on a topological reason nor on large curvature (sectional curvature decays almost as $d(x, 0)^{-2}$ ).

The metric on $\mathbf{R}^{4}$ will be given explicitly in terms of two functions $r(t), R(t)$ where $t$ is radial distance from $0 \in \mathbf{R}^{4}$ and

$$
\begin{aligned}
r(t) & := \begin{cases}\sin t \cdot \cos t & \text { for } t \leq t_{0}, \\
\sin t_{0} \cdot \cos t_{0} \cdot\left[1+\left(t-t_{0}\right) \cdot \alpha^{-1 / 2}\right]^{\alpha-1} & \text { for } t \geq t_{0},\end{cases} \\
R(t) & := \begin{cases}\sin t & \text { for } t \leq t_{0}, \\
\sin t_{0} \cdot\left[1+\left(t-t_{0}\right) \cdot \alpha^{-1 / 2}\right)^{\alpha} & \text { for } t \geq t_{0},\end{cases}
\end{aligned}
$$

with $\alpha \in\left(\frac{2}{3}, 1\right)$ to make Ric $>0$ and $\tan t_{0}=\alpha^{-1 / 2}$ to make both functions $C^{1}$ at $t_{0}$. Below (4.4) a small change is described to smooth the second derivatives at $t_{0}$. The ball of radius $t_{0}$ around $0 \in \mathbf{R}^{4}$ is isometric to a ball in $\mathbf{C} P^{2}$. For large $t$ sectional curvatures will be shown to decay as $t^{-2 \alpha}$.

Let $I, J, K$ be three pairwise orthogonal complex structures for $\mathbf{R}^{4}$. Define the corresponding Killing fields $X_{i}$ and orthogonal fields $E_{i}, N$ by

$$
\begin{aligned}
& X_{1}(p):=I \cdot p, \quad X_{2}(p):=J \cdot p, \quad X_{3}(p):=K \cdot p, \quad p \in \mathbf{R}^{4} ; \\
& E_{i}(p):=\frac{X_{i}}{\left\langle X_{i}, X_{i}\right\rangle}(p), \quad(i=1,2,3), \quad N(p):=\frac{p}{|p|}, \quad 0 \neq p \in \mathbf{R}^{4} .
\end{aligned}
$$

Put $R_{1}(t)=r(t), R_{2}(t)=R_{3}(t)=R(t)$ and define a Riemannian metric on $\mathbf{R}^{4}$ such that $|N|_{g}=1$ and $\left|X_{i}(p)\right|_{g}=R_{i}(|p|)$ :

$$
g(p)(U, V):=\langle U, N\rangle \cdot\langle N, V\rangle+\sum_{i} R_{i}^{2}(|p|) \cdot\left\langle U, E_{i}\right\rangle \cdot\left\langle E_{i}, V\right\rangle .
$$

Properties of the metric (4.0). The metric is complete since the rays from 0 remain geodesics of infinite length. $X_{1}$ is a Killing field also for the new metric since 
rotation $\exp (\varphi \cdot I)$ of $\mathrm{R}^{4}$ rotates $E_{2}$ to $E_{3}$ to $-E_{2}$, hence leaves the metric invariant $\left(R_{2}(t)=R_{3}(t)\right)$. Moreover the reflection of $\mathbf{R}^{4}$ which has as (+1)-eigenspace $\operatorname{span}\left\{N(p), E_{i}(p)\right\}$ sends $E_{i}$ to $E_{i}$ and $E_{j}, E_{k}$ to $-E_{j},-E_{k}$ and therefore also is an isometry for the new metric $(i=1,2,3)$. The fixed point sets of these reflections $(=2$-planes through 0$)$ are totally geodesic subspaces; their metric and curvature in polar coordinates can be read off directly:

$$
\begin{aligned}
& d t^{2}+R_{i}^{2}(t) d \varphi^{2} . \\
& \tilde{K}\left(E_{i}, N\right)=-\ddot{R}_{i}(t) / R_{i}(t), \quad \sim t^{-2} \text { as } t \rightarrow \infty .
\end{aligned}
$$

For $i=1$ ("Hopf planes") we have totally geodesic

subspaces whose injectivity radius goes to zero as $t^{\alpha-1}$.

The induced metrics on the distance spheres around 0 are Berger metrics; we reduce all curvature computations to known properties of Berger spheres which we quote from $[\mathbf{B K}]$ :

(BS1) The Ricci tensor is diagonal for the basis $\left\{E_{1}, E_{2}, E_{3}\right\}$.

(BS2) The sectional curvatures $K_{i j}$ are

$$
\begin{aligned}
& K\left(E_{1} \wedge E_{2}\right)=K\left(E_{1} \wedge E_{3}\right)=r^{2} \cdot R^{-4}, \\
& K\left(E_{2} \wedge E_{3}\right)=\left(4 R^{2}-3 r^{2}\right) \cdot R^{-4}
\end{aligned}
$$

and more precisely

$$
R\left(E_{i}, E_{k}\right) E_{k}=K_{i k} \cdot\left|E_{k}\right|^{2} \cdot E_{i} .
$$

Because of the totally geodesic 2-planes we also know that $E_{1}, E_{2}, E_{3}$ are the eigenvectors of the shape operators of the distance spheres around 0 ! We compute the eigenvalues from (4.1):

$$
\begin{aligned}
& \tilde{D}_{E_{1}} N=\frac{\dot{r}}{r} \cdot E_{1}=-\kappa_{1} \cdot E_{1}, \quad \tilde{D}_{E_{j}} N=\frac{\dot{R}}{R} E_{j}=-\kappa_{j} \cdot E_{j} \quad(j=2,3) ; \\
& \kappa_{i} \sim t^{-1} \quad \text { as } t \rightarrow \infty(i=1,2,3) .
\end{aligned}
$$

Now the Gauss equations and (BS2) give the following curvatures of the metric (4.0):

$$
\begin{array}{ll}
\tilde{K}\left(E_{1} \wedge E_{2}\right)=\tilde{K}\left(E_{1} \wedge E_{3}\right)=\frac{r^{2}}{R^{4}}-\frac{\dot{R}}{R} \cdot \frac{\dot{r}}{R} & \sim t^{-2} \quad \text { as } t \rightarrow \infty, \\
\tilde{K}\left(E_{2} \wedge E_{3}\right)=\frac{4 R^{2}-3 r^{2}}{R^{4}}-\left(\frac{\dot{R}}{R}\right)^{2} & \sim t^{-2 \alpha} \quad \text { as } t \rightarrow \infty .
\end{array}
$$

We continue to use the totally geodesic 2-planes. If $W$ is any normal to a totally geodesic submanifold and $U, V$ are tangents then

$$
\tilde{R}(U, V) W \text { is normal to the submanifold. }
$$

In our case:

$$
\tilde{R}\left(E_{i}, N\right) E_{j} \perp E_{i}, N ;
$$

in particular $N$ is an eigenvector of Ric, hence with (4.1)

$$
\widetilde{\operatorname{Ric}}(N)=(-\ddot{r} / r-2 \ddot{R} / R) \cdot N \text {. }
$$


Consequently $\operatorname{span}\left\{E_{1}, E_{2}, E_{3}\right\}$ is an invariant subspace of Ric. Now (BS1), the diagonality of the shape operator relative to $\left\{E_{1}, E_{2}, E_{3}\right\}$ and the Gauss equations

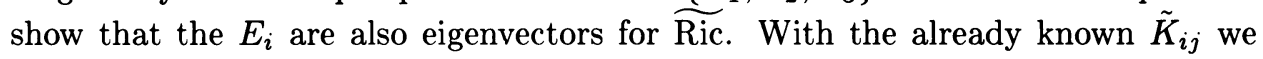
find:

$$
\begin{aligned}
\widetilde{\operatorname{Ric}}\left(E_{1}\right) & =\left(2 \cdot\left(\frac{r^{2}}{R^{4}}-\frac{\dot{R} \dot{r}}{R r}\right)-\frac{\ddot{r}}{r}\right) \cdot E_{1}, \\
\widetilde{\operatorname{Ric}}(E) & =\left(\frac{2\left(R^{2}-r^{2}\right)}{R^{4}}+\frac{2-\dot{R}^{2}}{R^{2}}-\frac{\dot{R} \dot{r}}{R r}-\frac{\ddot{R}}{R}\right) \cdot E \quad \text { for } E \perp E_{1}, N .
\end{aligned}
$$

Claim. The eigenvalues of Ric are $>0$. We need to check this only for $t>t_{0}$ :

$$
\begin{aligned}
& \lambda_{N}>0 \Leftrightarrow(1-\alpha) \cdot\left(\alpha-\frac{2}{3}\right)>0, \\
& \lambda_{E_{1}}>\lambda_{N} \text { since } \dot{R} \dot{r} / R r=\ddot{R} / R, \\
& \lambda_{E}>0 \text { since all terms in the sum are positive. }
\end{aligned}
$$

Smoothing of the upward jump of the second derivative of $r$ and $R$ at $t_{0}$ : Note that the second derivatives enter linearly into curvature formulas and we have open inequalities to satisfy. We therefore smooth in such a way that the second derivative stays between the second derivatives of the two functions to join and such that first derivatives and values are changed sufficiently little so that Ric $>0$ is not destroyed.

At this point the example is already relevant to the question (0.1). We improve it further by controlling the asymptotic decay of all sectional curvatures. The largest of those already computed is $\tilde{K}\left(E_{2} \wedge E_{3}\right) \sim t^{-2 \alpha}(4.2)$. We show that the remaining curvature tensor components decay faster.

From (BS2), the Gauss equations and the diagonality of the shape operator we have $g\left(\tilde{R}\left(E_{i}, E_{j}\right) E_{k}, E_{i}\right)=0$ or

$$
\tilde{R}\left(E_{i}, E_{j}\right) E_{k} \text { is proportional to } N \text { for }\{i, j, k\}=\{1,2,3\} \text {. }
$$

Since the Killing field $X_{1}$ rotates $E_{2}$ to $E_{3}$ to $-E_{2}$ we have together with the first Bianchi identity

$$
\tilde{R}\left(E_{1}, E_{2}\right) E_{3}=\tilde{R}\left(E_{1},-E_{3}\right) E_{2}=\frac{1}{2} \tilde{R}\left(E_{3}, E_{2}\right) E_{1} .
$$

Therefore it suffices to bound $g\left(\tilde{R}\left(E_{2}, E_{3}\right) N, E_{1}\right)$. Denote the covariant derivatives of $\left(\mathbf{R}^{4}, g\right)$ resp. of the Berger spheres by $\tilde{D}$ resp. $D$. Then, using the diagonality of the shape operator and $\{i, j, k\}=\{1,2,3\}$ :

$$
\tilde{D}_{E_{j}} N=-\kappa_{j} \cdot E_{j}, \quad \tilde{D}_{E_{i}} E_{j}=D_{E_{i}} E_{j}=g\left(D_{E_{i}} E_{j}, E_{k}\right) \cdot \frac{E_{k}}{g\left(E_{k}, E_{k}\right)} .
$$

Since $E_{1}$ is a Killing field on each distance sphere (around 0) we have

$$
g\left(D_{E_{2}} E_{3}, E_{1}\right)=-g\left(E_{3}, D_{E_{2}} E_{1}\right) \underset{(\text { Killing })}{=} g\left(E_{2}, D_{E_{3}} E_{1}\right)=-g\left(D_{E_{3}} E_{2}, E_{1}\right)
$$

and the derivative of this quantity tangential to a sphere is zero. We differentiate (4.6) and use (4.7) to get

$$
g\left(\tilde{R}\left(E_{2}, E_{3}\right) N, E_{1}\right)=\left(\kappa_{2}+\kappa_{3}-2 \kappa_{1}\right) \cdot g\left(D_{E_{2}} E_{1}, E_{3}\right) .
$$


Use once more that $E_{1}$ is a Killing field together with the constancy of (4.7): $R\left(E_{1}, E_{2}\right) E_{2} \underset{\text { (Killing) }}{=}-D_{E_{2}}\left(D_{E_{2}} E_{1}\right) \underset{(4.6,4.7)}{=}-g\left(E_{3}, E_{3}\right)^{-1} \cdot g\left(D_{E_{2}} E_{1}, E_{3}\right) \cdot D_{E_{2}} E_{3}$ or with (BS2):

$$
\left|D_{E_{2}} E_{1}\right|_{g}^{2}=g\left(R\left(E_{1}, E_{2}\right) E_{2}, E_{1}\right)=r^{2} R^{-4} \cdot\left|E_{1}\right|^{2} \cdot\left|E_{2}\right|^{2} .
$$

We use this and the $t^{-1}$-decay of the $\kappa_{i}$ to get from (4.8) the desired asymptotic behaviour:

$$
g\left(\tilde{R}\left(E_{2}, E_{3}\right) N, E_{1}\right) \sim t^{-\alpha-2} \cdot\left|E_{1}\right| \cdot\left|E_{2}\right| \cdot\left|E_{3}\right| .
$$

For given $\varepsilon$ one can make $t$ so large that curvature effects are arbitrarily small in an $\varepsilon$-ball. These balls then look approximately like the product of a 3-dim flat disc of radius $\varepsilon$ with a circle of length $2 \cdot$ inj-rad, i.e.

$$
\operatorname{vol}\left(B_{\varepsilon}\right) \approx \frac{4 \pi}{3} \varepsilon^{3} \cdot 2 \cdot \operatorname{inj}-\operatorname{rad} \approx \text { const } \cdot \varepsilon^{3} \cdot t^{\alpha-1} .
$$

\section{REFERENCES}

[A1] A. D. Aleksandrov, Die innere Geometrie der konvexen Flächen, Akademieverlag, Berlin, 1955.

[BK] J.-P. Bourguignon and H. Karcher, Curvature operators: Pinching estimates and geometric examples. Ann. Sci. Ecole Norm. Sup. (4) 11 (1978), 71-92.

[BZ] Yu. D. Burago and V. A. Zalgaller, Convex sets in Riemannian spaces of nonnegative curvature, Russian Math. Surveys 32:3 (1977), 1-57.

[CG] J. Cheeger and D. Gromoll, On the structure of complete manifolds of nonnegative curvature, Ann. of Math. 96 (1972), 413-443.

[Cr] C. Croke, Some isoperimetric inequalities and eigenvalue estimates, Ann. Sci. Ecole Norm. Sup. (4) 13 (1980), 419-435.

[GM] D. Gromoll and W. Meyer, On complete open manifolds of positive curvature, Ann. of Math. 90 (1969), 75-90.

[Gr] M. Gromov, Isoperimetric inequalities in Riemannian manifolds, pp. 114-129 in V. D. Milman and G. Schechtman, Asymptotic Theory of Finite Dimensional Normed Spaces, Lecture Notes in Math., vol. 1200, Springer, 1986.

[Sh] V. A. Sharafutdinov, The radius of injectivity of a complete open manifold of nonnegative curvature, Dokl. Akad. Nauk SSSR 231 (1976), 46-48.

Institut des hautes Etudes Scientifiques, 35, Route De Chartres, 91440 Bures-Sur-YvetTE, FRANCE

Current address (Christopher B. Croke): Department of Mathematics, University of Pennsylvania, Philadelphia, Pennsylvania 19104-6395

Current address (Hermann Karcher): Universität Bonn, Bonn, Federal Republic of Germany 